\title{
Caracterização física e mecânica de um compósito de polipropileno reciclado e farinha de madeira sem aditivos
}

\author{
Physical and mechanical characterization \\ of a recycled polypropylene and wood \\ flour without additives
}

Rosane Battistelle, Natalia Martin Viola,

Barbara Stolte Bezerra, Ivaldo De Domenico Valarelli,

Universidade Estadual Paulista "Júlio de Mesquita Filho" (UNESP)

Faculdade de Engenharia de Bauru (FEB), Programa de Pós-Graduação em Engenharia de Produção

Av. Eng. Luiz Edmundo C. Coube, 14-01, Bairro Vargem Limpa, Bauru, São Paulo, Brasil. Telefone (14) 3103-6000.

e-mail: rosane@feb.unesp.br; nataliamviola@hotmail.com

\section{RESUMO}

Compósitos de matriz polimérica reciclada reforçados com farinha de madeira podem ser uma alternativa viável para a substituição da madeira e de polímeros virgens em materiais empregados em pisos, batentes de portas, janelas e revestimentos externos. O objetivo desta pesquisa foi determinar alguns parâmetros físicos e mecânicos de compósito com farinha de madeira de Pinus taeda e elliotti e polipropileno reciclado, sem o emprego de compatibilizantes ou aditivos. Os compósitos foram separados em quatro traços, sendo eles: $100 \%$ polipropileno, $90 \%$ polipropileno com $10 \%$ de farinha de madeira, $80 \%$ de polipropileno com $20 \%$ de farinha de madeira e 70\% de polipropileno com $30 \%$ de farinha de madeira. Para caracterização do compósito foram utilizadas as normas ASTM D-638-10, ASTM D256-00, ASTM D570-98, ASTM D1238-10 e ASTM G-155-05 e também a análise de superfície por microscopia eletrônica de varredura. Os ensaios de estabilidade dimensional apresentaram resultados satisfatórios. Mesmo o compósito com maior porcentagem de farinha de madeira (30\%) apresentou um melhor índice de fluidez de 10,0MFI, sendo considerado compatível com os índices para PP (polipropileno) virgem da norma ASTM D-1238-10. A inclusão de farinha de madeira (FM) proporcionou a obtenção de compósitos com boas características mecânicas que podem ser aplicados na fabricação de diferentes materiais, empregados especificamente em ambientes externos.

Palavras-chave: farinha de madeira, polipropileno reciclado, compósito, aproveitamento de resíduos.

\begin{abstract}
Recycled polymer matrix composites reinforced with wood flour can be a viable alternative for the replacement of wood and virgin polymers in materials used in floors, door frames, windows and external cladding. The objective of this research was to determine some physical and mechanical parameters of composite made with Pinus taeda and elliottii wood flour (WF) and recycled polypropylene (PP), without the use of compatibilizers or additives. The composites were separated into four traits, namely $100 \%$ PP, 90\% PP with $10 \%$, WF $80 \%$ PP with $20 \% \mathrm{WF}$ and $70 \%$ PP with $30 \% \mathrm{WF}$. The characterization of the composite followed the standards ASTM D-638-10, ASTM D256-00, ASTM D570 -98, ASTM D1238 -10 and ASTM G 155-05, it was also employed the surface analysis by scanning electron microscopy. The dimensional stability tests showed satisfactory results. Even the composite with a higher percentage of wood flour (30\%) had a flow index of $10 \mathrm{MFI}$, considered compatible with that observed for PP (polypropylene) virgin by standard ASTM D 1238-10. The inclusion of wood flour (FM) afforded composites with good mechanical characteristics which can be applied in manufacture of different materials, specifically employed outdoors.
\end{abstract}

Keywords: Wood flour, recycle polypropylene, composites, waste recovery.

\section{INTRODUÇÃO}

Com a globalização e o crescimento populacional, existe a necessidade de se estudar o desenvolvimento de 
novos produtos a partir de seus resíduos (em diferentes formulações) com a inserção, ou não, de materiais poliméricos pós-consumo, de forma a minimizar o emprego da matéria prima virgem e reduzir o descarte de materiais nobres.

Uma aplicação atual para o polipropileno de embalagens do tipo PET e dos resíduos proveniente do beneficiamento da madeira que vem ganhando espaço no mercado internacional é a sua utilização como reforço na fabricação de compósitos. Estes materiais são constituídos por uma fase polimérica (matriz), e outra, como fase de reforço, que pode ser aplicada na forma de fibras.

Para MENEGHETTI et al [1], o uso de novas formulações fibra-matriz devem não somente visar à redução dos custos, mas também obter combinações fibras-matriz com comportamentos mecânicos apropriados e de boa compatibilidade, além de buscar uma melhoria na resistência ao impacto e às falhas de acabamento.

De acordo com NEWELL [2], o termo compósito é definido como um material formado por dois ou mais componentes, que se misturam para originar um terceiro com propriedades diferentes, sendo classificados em três categorias: reforçados com fibras, particulados e laminados.

Os compósitos de plástico-madeira, também chamados de WPC (Wood-plastic composites), começaram a ser produzidos na Europa e nos Estados Unidos, sendo caracterizados como materiais leves e de fácil moldagem, permitindo assim, formas complexas sem emendas, abrangendo uma grande variedade de utilizações. Uma das vantagens mais evidentes desses materiais é a possibilidade de utilização de resíduos de outros processos produtivos como reforço no compósito. Desta forma, podem-se utilizar resíduos provenientes do corte de madeira para produção do compósito, reduzindo os custos do produto final e o desperdício [3].

Segundo RODOLFO e JOHN [4], uma das alternativas para produção do WPC é a utilização do PVC com farinha de madeira. As aplicações em produtos externos como decks apresentam durabilidade superior à da madeira, fazendo com que os componentes necessitem de menos manutenção quando expostos às intempéries. Além disso, os produtos fabricados são muito versáteis quanto ao design e acabamento, o que propicia a criação de peças inovadoras.

Os compósitos com resíduos de madeira podem ser combinados com polímeros (com baixa temperatura de processamento) como o polipropileno (PP), poliésteres (PE) e policloreto de vinila (PVC), de forma a não danificar as partículas de madeira durante a fabricação do compósito [5]. As fibras de madeira utilizadas em reforços e cargas para plásticos, também podem substituir materiais de alto custo, como o carbonato de cálcio e a fibra de vidro. Nos WPC's, o plástico melhora a resistência à umidade e ao ataque de insetos e fungos. Assim, as boas características de superfície, resistência e baixo custo são atrativos para o desenvolvimento de pesquisas com compósitos e suas possíveis aplicações de produtos inovadores.

O primeiro compósito plástico-madeira, segundo CLEMONS [3], foi patenteado em 1971, na Itália, por um processo de extrusão que utilizou $50 \%$ de farinha de madeira e 50\% de polipropileno, sendo extrudados em forma de lâminas para a indústria automobilística. Já para o processo de injeção, uma nova tecnologia de processamento de compósitos com fibras naturais e PP foi desenvolvida em 2003, para a indústria automobilística, por possuir boas propriedades mecânicas, além de menor densidade e peso [3]. Na atualidade, podemse produzir compósitos de plástico-madeira, em diferentes traços, em formas de chapas, extrudados ou injetados. No entanto NAJAFI [6] aponta que há viabilidade no uso comercial do compósito plástico-madeira, mas que há poucas e limitadas pesquisas a respeito, principalmente no que fala sobre propriedades físicas e mecânicas dos WPC's utilizando polímeros reciclados.

Em relação à viabilidade econômica, pode-se mencionar que nas indústrias de plásticos dos EUA, no ano 2000, o valor estimado estava em 9,2 bilhões de dólares, dos quais 7\% foram estimados para as fibras biológicas, sendo a maioria delas extraídas da madeira, além do emprego de uma pequena parcela de outras fibras naturais, como linho ou palha de trigo. O preço médio por quilo de plástico reforçado com fibra, em 2000, era de $\mathrm{U} \$ 0,90$ para fibra de vidro, $\mathrm{U} \$ 0,20$ para fibras naturais (não a madeira), $\mathrm{U} \$ 0,10$ para fibra de madeira e o carbonato de cálcio em U $\$ 0,70$ [7]. Desta forma, fica perceptível a viabilidade da utilização de resíduos de madeira e plástico reciclado como componente em compósitos e como alternativa na fabricação de novos produtos.

Muitas pesquisas estão sendo realizadas sobre WPC's, sendo que alguns autores, como STARK [8] e JOSEPH et al [9], que estudaram a influência da umidade nos compósitos,verificaram que o maior percentual de fibras influi na absorção de água. PENDLETON et al. [10] produziram um compósito com 50\%FM e verificaram através de ensaios mecânicos, que com o aumento do módulo tensão em contrapartida, a resistência à tração e impacto diminuíram para este percentual de fibras. STARK et al. [11] analisaram as características do WPC, em diferentes composições, com relação ao seu comportamento diante do fogo e calor. 
Neste trabalho foram produzidos compósitos com farinha de madeira de Pinus taeda e elliotti com polipropileno reciclado, sem o emprego de compatibilizantes ou aditivos. Os compósitos foram separados em quatro traços, sendo eles: $100 \%$ polipropileno, $90 \%$ polipropileno com $10 \%$ de farinha de madeira, $80 \%$ de polipropileno com $20 \%$ de farinha de madeira e $70 \%$ de polipropileno com $30 \%$ de farinha de madeira.

\section{MATERIAIS E MÉTODO}

A produção do compósito plástico-madeira envolveu formulações com madeira e o termoplástico reciclado. O compósito de polipropileno reciclado foi elaborado sem adição de nenhum tipo de aditivo ou compatibilizante e caracterizado nas suas propriedades físicas e mecânicas como resistência ao impacto, alongamento e tração, absorção de água e índice de fluidez.

\subsection{Materiais}

A farinha de madeira (FM) usada para a produção do compósito plástico-madeira proveniente da mistura das espécies Pinus taeda e Pinus elliotti foi fornecida pela indússtria Pinhopó Moagem de Madeira, localizada em Ponta Grossa-PR-Brasil. O material foi obtido por meio da moagem de resíduos de madeira e não continham nenhuma impureza, possuindo uma coloração clara, com granulometria uniforme (Figura 1(a)). A madeira do tipo Pinus foi escolhida por ser uma das madeiras mais plantadas nas regiões Sul e Sudeste do Brasil, além de ser utilizada na extração de resinas [12].Este foi um ponto importante para a pesquisa já que não foi utilizado nenhum compatibilizante para a aglutinação do polímero com a madeira. Antes do início do processamento do compósito, a madeira necessitou permanecer em estufa, por um período de $3 \mathrm{~h}$ com uma temperatura de $60^{\circ} \mathrm{C}$.

Nesta pesquisa foi escolhido o polímero polipropileno (PP) por ser um polímero totalmente reciclável e possuir uma temperatura de amolecimento compatível para a produção do WPC, proveniente de uma Recicladora de Plásticos localizada na cidade de Bauru-SP, Brasil. A matéria-prima estava em forma de pellets, porém sem homogeneidade e com algumas impurezas (Figura 1(b)). O material foi lavado (Figura 1(c)) e seco em estufa por $24 \mathrm{~h}$ à $90^{\circ} \mathrm{C}$ antes da fabricação do compósito plástico-madeira.

Em seguida, teve início o processo de extrusão do material para a formação dos pellets, matéria prima objeto do estudo, sendo que a extrusora estava programada para as zonas de aquecimento, sempre com a temperatura da água à $25^{\circ} \mathrm{C}$, da seguinte forma: $\mathrm{Z} 1$ à $110^{\circ} \mathrm{C} ; \mathrm{Z} 2$ à $150^{\circ} \mathrm{C} ; \mathrm{Z3}$ à $170^{\circ} \mathrm{C} ;$ de $\mathrm{Z4}$ a $\mathrm{Z6}$ à $195^{\circ} \mathrm{C} ; \mathrm{Z} 8$ a $\mathrm{Z10}$ à $190^{\circ} \mathrm{C} ; \mathrm{Z} 11$ à $200^{\circ} \mathrm{C}$; sendo as velocidade de extrusão de $50 \mathrm{~kg} / \mathrm{h}$ para $100 \% \mathrm{PP}$ e $90 \% \mathrm{PP}+10 \% \mathrm{FM} \mathrm{e} 20 \mathrm{~kg} / \mathrm{h}$ para $80 \% \mathrm{PP}+20 \% \mathrm{FM}$ e $70 \% \mathrm{PP}+30 \mathrm{FM}$.

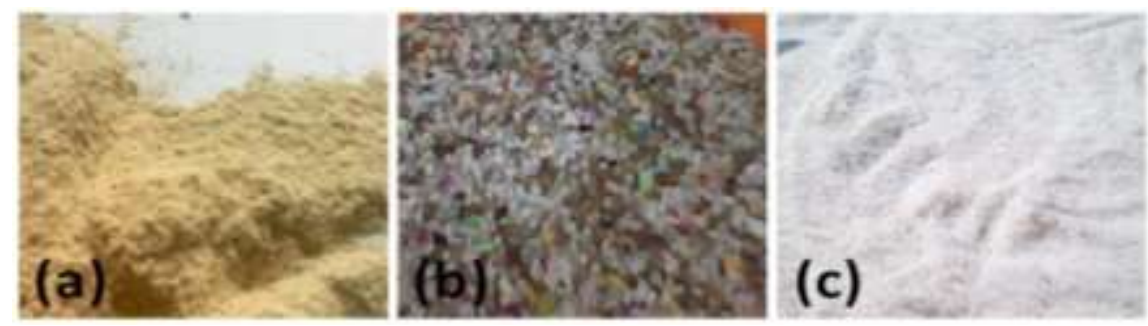

Figura 1: Amostras da (a)farinha de madeira e do PP (b) antes e (c)após lavagem.

\subsection{Método}

Os corpos de prova foram produzidos e ensaiados com base nas normas ASTM-D-638-10 [13], ASTM D570-98 [14], ASTM D-1238-10 [15] e ASTM D-256-00 [16]. Para tanto, foram desenvolvidos quatro traços, com peso total de $5 \mathrm{~kg}$ de material para cada, conforme os dados da Tabela 1. O diagrama da produção do compósito é mostrado na Figura 2.

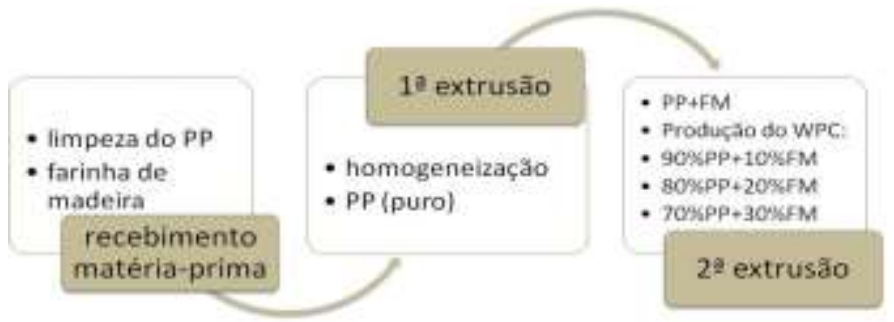


Figura 2: Diagrama da produção do compósito.

\subsection{Produção do compósito}

A produção do compósito plástico-madeira foi feita no Laboratório de Biocycle, da Universidade Federal de São Carlos-UFSCar e no Laboratório de Anelasticidade e Biomateriais da Faculdade de Ciências, no Laboratório de Processamento da Madeira da Faculdade de Engenharia, ambos da UNESP - Bauru.

Tabela 1: Traços dos compósitos e programação da injetora para cada formulação.

\begin{tabular}{|c|c|c|c|c|c|}
\hline Traço & $\begin{array}{l}\text { Quantidade de } \\
\text { material }\end{array}$ & $\begin{array}{c}\% \\
\text { de Material } \\
\end{array}$ & $\begin{array}{c}\text { Temperatura das } 4 \text { zonas } \\
\text { de aquecimento }\end{array}$ & $\begin{array}{l}\text { Velocidade } \\
\text { de injeção }\end{array}$ & $\begin{array}{l}\text { Temperatura } \\
\text { da água }\end{array}$ \\
\hline 1 & $5 \mathrm{~kg}$ & $100 \% \mathrm{PP}$ & $\begin{array}{c}\mathrm{z} 1-200^{\circ} \mathrm{C} ; \mathrm{z} 2-199,5^{\circ} \mathrm{C} \\
\mathrm{z} 3-194,8^{\circ} \mathrm{C} ; \mathrm{z} 4-191^{\circ} \mathrm{C}\end{array}$ & $15 \mathrm{seg} / \mathrm{bar}$ & $30^{\circ} \mathrm{C}$ \\
\hline 2 & $4,5 \mathrm{~kg} \mathrm{PP}+0,5 \mathrm{~kg} \mathrm{FM}$ & $90 \% \mathrm{PP}+10 \% \mathrm{FM}$ & $\begin{array}{l}\mathrm{z} 1-190,1^{\circ} \mathrm{C} ; \mathrm{z} 2-189,9^{\circ} \mathrm{C} \\
\mathrm{z} 3-185,2^{\circ} \mathrm{C} ; \mathrm{z} 4-159,8^{\circ} \mathrm{C}\end{array}$ & $20 \mathrm{seg} / \mathrm{bar}$ & $30^{\circ} \mathrm{C}$ \\
\hline 3 & $04 \mathrm{~kg} P \mathrm{PP}+01 \mathrm{~kg} F M$ & $80 \% \mathrm{PP}+20 \% \mathrm{FM}$ & $\begin{array}{l}\mathrm{z} 1-190,1^{\circ} \mathrm{C} ; \mathrm{z} 2-189,9^{\circ} \mathrm{C} \\
\mathrm{z} 3-185,2^{\circ} \mathrm{C} ; \mathrm{z} 4-159,8^{\circ} \mathrm{C}\end{array}$ & 20seg/bar & $30^{\circ} \mathrm{C}$ \\
\hline 4 & $3,5 \mathrm{~kg} \mathrm{PP}+1,5 \mathrm{~kg} \mathrm{FM}$ & $70 \% \mathrm{PP}+30 \% \mathrm{FM}$ & $\begin{array}{l}\mathrm{z} 1-190,1^{\circ} \mathrm{C} ; \mathrm{z} 2-189,9^{\circ} \mathrm{C} ; \\
\mathrm{z} 3-185,2^{\circ} \mathrm{C} ; \mathrm{z} 4-159,8^{\circ} \mathrm{C}\end{array}$ & 20seg/bar & $30^{\circ} \mathrm{C}$ \\
\hline
\end{tabular}
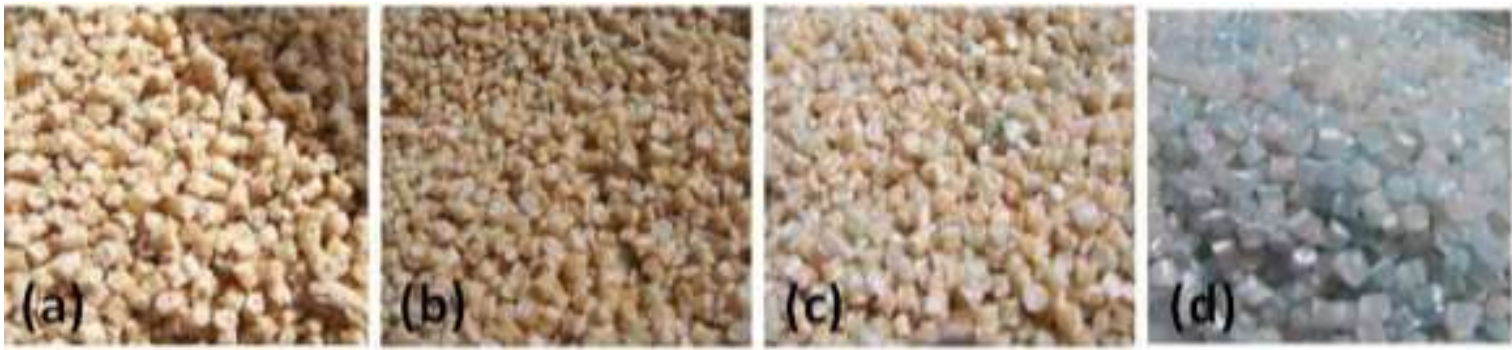

Figura 3: Pellets do compósito plástico-madeira em (a) 70\%PP+30\% FM,(b) 80\%PP+20\% FM, (c)90\%PP+10\% FM e (d) $100 \% \mathrm{PP}$, respectivamente.

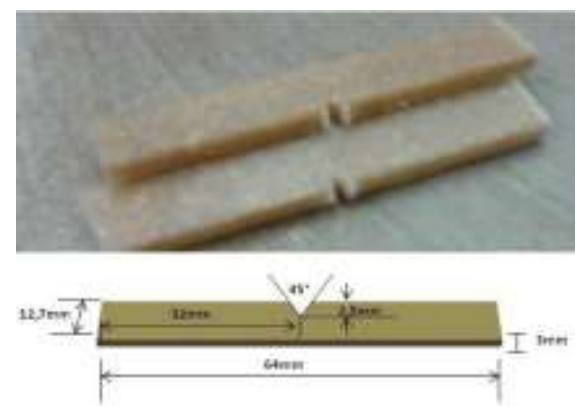

(a)
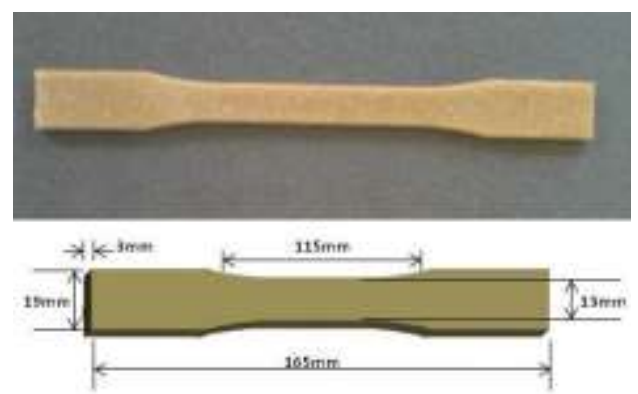

(b)

(c)

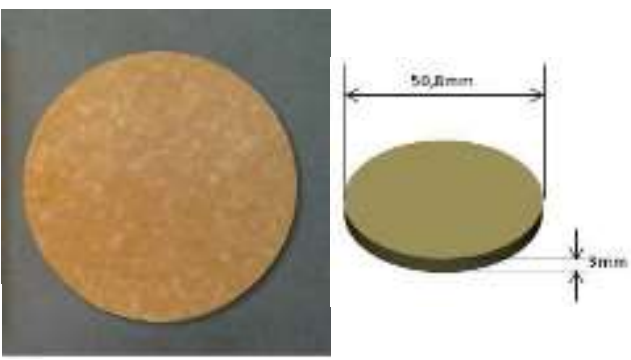


Figura 4: Detalhe dos corpos de prova separados para os diferentes ensaios (a) corpo de prova (charpy) para o ensaio de impacto; (b) corpo de prova para o ensaio de tração; (c) corpo de prova para o ensaio de absorção de água e inchamento em espessura.

Após a extrusão para homogeneização do PP puro (Figura 2(d)), foi realizada a pesagem com cálculo de percentual para a produção do compósito, juntamente com a farinha de madeira. $\mathrm{O}$ material foi colocado em recipientes e misturado por aproximadamente dois minutos, para uma melhor aglutinação das partes. Em seguida, teve início o processo de extrusão do compósito (união do PP com a FM em forma de pellets), utilizando-se de uma extrusora de rosca dupla com onze zonas de aquecimento, com variação de temperaturas entre $110^{\circ} \mathrm{C}$ (para zona 01 ) e $200^{\circ} \mathrm{C}$ (zona 11), com velocidade de extrusão a $20 \mathrm{~kg} / \mathrm{h}$ para $70 \% \mathrm{PP}+30 \% \mathrm{FM}$ (Figura 3(a)) e $80 \%$ PP+20\%FM (Figura 3(b)), e 50kg/h para 90\%PP+10\%FM (Figura 3(c)) e 100\%PP (Figura $3(\mathrm{~d}))$.

Após o processo, as formulações ficaram em estufa por um período de $24 \mathrm{~h}$ em uma temperatura de $60^{\circ} \mathrm{C}$. E, após a secagem, o processo de injeção (Injetora Romi Pratica, Modelo 130) foi iniciado de acordo com as condições descritas na Tabela 1 para a confecção dos corpos de prova, segundo a norma ASTM D-570-98 [14].

Os corpos de prova foram cortados das chapas seguindo as dimensões e descrições da norma pertinente ao ensaio de impacto (Figura 4(a)), tração (Figura 4(b)), e absorção de água e inchamento em espessura (Figura $4(\mathrm{c}))$.

\section{RESULTADOS E DISCUSSÕES}

Os resultados para cada traço são apresentados na Tabela 2, com a indicação dos parâmetros base de especificações da norma [13] para o PP virgem, pois não há especificação para os compósitos fabricados com material reciclado de PP.

Tabela 2: Resumo dos dados obtidos nos ensaios físicos e mecânicos.

\begin{tabular}{|c|c|c|c|c|c|}
\hline \multirow{2}{*}{ Ensaios } & \multicolumn{4}{|c|}{ Traços } & \multirow{2}{*}{ Parâmetros base de especificações } \\
\cline { 2 - 5 } & Controle & \multicolumn{3}{|c|}{ Compósitos } & \\
\hline $\boldsymbol{\sigma}_{\mathbf{Y}}$ & $22,12 \pm 0,63$ & $21,64 \pm 0,17$ & $20,97 \pm 0,25$ & $20,13 \pm 0,15$ & ASTM D-638 [3] (PP virgem 31.0-37.2 MPa) \\
\hline $\boldsymbol{\varepsilon}_{\boldsymbol{E}}$ & $9,17 \pm 0,17$ & $6,05 \pm 0,15$ & $3,94 \pm 0,13$ & $2,62 \pm 0,07$ & ASTM D-638 [3] (PP virgem 13\%) \\
\hline $\boldsymbol{E}$ & $1,00 \pm 0,04$ & $1,39 \pm 0,02$ & $1,79 \pm 0,05$ & $2,17 \pm 0,04$ & ASTM D-638 [3] (PP virgem 1.14-1.55 GPa) \\
\hline$\sigma_{\mathrm{T}}$ & $18,80 \pm 0,55$ & $20,28 \pm 0,59$ & $20,59 \pm 0,33$ & $20,06 \pm 0,15$ & ASTM D-638 [3] (PP virgem: $3,63 \mathrm{MPa})$ \\
\hline $\boldsymbol{\varepsilon}_{\mathbf{U}}$ & $367,01 \pm 17,03$ & $9,90 \pm 1,37$ & $4,59 \pm 0,31$ & $2,76 \pm 0,15$ & ASTM D-638 [3] (PP virgem: 8,79\%) \\
\hline $\boldsymbol{I}_{\boldsymbol{s}}$ & $75,55 \pm 7,55$ & $41,06 \pm 5,20$ & $33,94 \pm 2,10$ & $34,94 \pm 1,44$ & ASTM D-256 [4] (PP virgem: 2,66J/m) \\
\hline Aa $^{*}$ & 0,24 & 0,49 & 1,01 & 1,02 & YAMAJI [19] (0,3-1,1\%) \\
\hline Ie* $^{*}$ & $1,10 \pm$ & 1,47 & 2,22 & 2,95 & YAMAJI [19] (0,2-0,8\%) \\
\hline MFI* & $30,00 \pm$ & 21,80 & 16,50 & 10,00 & ASTM D-1238 [5] (PP virgem: 2.23-32.8 g/10min) \\
\hline
\end{tabular}

* Não foi calculado desvio padrão para estes ensaios

** Este ensaio foi realizado com $100 \mathrm{~g}$ do material e apenas uma vez, por isso, não possui desvio-padrão.

Legenda:

\begin{tabular}{|l|l|}
\hline & Próximos aos valores normalizados \\
\hline & Acima dos valores normalizados \\
\hline & Abaixo dos valores normalizados \\
\hline & Dentro dos valores normatizados \\
\hline
\end{tabular}

Onde: $\boldsymbol{\sigma Y}$ : tensão no escoamento (MPa); EE: deformação no escoamento (\%); E: módulo de elasticidade (GPa); $\boldsymbol{\sigma}$ T: tensão na ruptura (MPa); $\boldsymbol{\varepsilon U}$ : deformação na ruptura (\%); Is: Resistência ao impacto (J/m); Aa: absorção de água (\%); Ie: inchamento em espessura (\%); e MFI: índice de fluidez (g/10min). 
Conforme os dados da Tabela 2, no ensaio de absorção de água foi possível notar no Traço 4 $(70 \% \mathrm{PP}+30 \% \mathrm{FM})$ valores mais elevados, significando que, quanto mais partículas de madeira na composição, maior o inchamento em espessura e a absorção de água. Na pesquisa de YAMAJI [17] foi verificado que os compósitos com 30\%FM também apresentaram maior absorção de água, com um total de 1,1\% para o compósito $70 \% \mathrm{PP}+30 \% \mathrm{FM}$, e que, a absorção de água é menor nos compósitos com agentes compatibilizantes, com um total de $0,7 \%$ para o compósito com $66,25 \% \mathrm{PP}+30 \% \mathrm{FM}+3 \% \mathrm{MA}+0,75 \% \mathrm{BP}$ (MA-anidrido maleico e BP-peróxido de benzoíla).

Dos compósitos, o traço T2 com 10\% FM obteve melhores resultados em tensão e deformação no escoamento enquanto o traço $\mathrm{T} 3$ obteve melhor resultado em tensão na ruptura. $\mathrm{O}$ traço $\mathrm{T} 4$ atingiu o maior resultado no módulo de elasticidade longitudinal. O trabalho de HILLIG [18] obteve em seus compósitos elaborados com $70 \% \mathrm{PE}+30 \% \mathrm{FM}$ (polietileno), o resultado de 20,14 MPa, na tensão de tração no escoamento, e desvio padrão em 0,80 MPa. Comparando com os resultados obtidos neste trabalho, no traço T4 com 70\%PP+30\%FM, pode-se verificar valor muito semelhante $(20,13 \mathrm{MPa})$, sendo que o desvio padrão é bem menor, em 0,15 MPa, sugerindo que a confecção dos corpos de prova se deu de forma mais uniforme.

Para os ensaios de tração, pode-se verificar que o Traçol de controle (com 100\%PP reciclado) teve maior índice de deformação na ruptura, com diferenças de desempenho nas amostras com desvio padrão em 17\%, uma vez que os corpos de prova não se romperam e chegaram no limite do teste, o qual teve que ser interrompido.

Nos ensaios de impacto, os corpos de prova do controle em 100\%PP (T1) não se romperam, com uma resistência de 75,55 J/m, sendo superior ao obtido por CORREA et al. [19] em seu controle de 100\%PP-Ho (polipropileno homopolímero puro). Tal resultado é interessante, uma vez que, nesta pesquisa, foi utilizado um polímero reciclado sem compatibilizantes, instigando para pesquisas futuras, análises químicas do material. No entanto, a resistência ao impacto para o compósito 70\% PP+30\%FM (T4) foi de 34,94 J/m, um resultado semelhante ao obtido por CORREA et al. [19], de 36,3 J/m para o compósito $60 \% \mathrm{PP}+30 \% \mathrm{FM}+10 \% \mathrm{PP}-\mathrm{MA}$ (PP modificado com anidrido maleico) visto que o pesquisador fez uso de agentes compatibilizantes. Foi possível verificar nos corpos de prova 90\% PP+10\%FM, que a resistência ao impacto foi de 41,06 J/m, a maior dos compósitos, e o compósito que apresentou menor desvio padrão foi o traço T4 (70\%PP+30\%FM), com um índice de $\pm 1,44 \mathrm{~J} / \mathrm{m}$, sendo inferior ao compósito $70 \% \mathrm{PP}+30 \% \mathrm{FM}$ com $\pm 2,0 \mathrm{~J} / \mathrm{m}$ obtido por CORREA et al. [19].

No ensaio de índice de fluidez, averiguou-se que, quanto maior o percentual de farinha de madeira na composição, menor o índice de fluidez. No entanto, o valor MFI para o compósito do traço T4 (70\%PP+30\%FM), é semelhante ao índice de fluidez, encontrado no PP virgem de 9,24 a 12,2 MFI, obtido por CARASCHI e LEÃO [20], com o destaque que o polímero usado nesta pesquisa é reciclado.

Foi observado o fator umidade como limitante dos processos de injeção, pois pode alterar a programação dos equipamentos e os resultados finais, sendo de extrema importância a secagem prévia do compósito em estufa.

Foi possível também a utilização da injetora com ajustes para o polipropileno virgem, sem modificações quanto ao processo tradicional de fabricação. Somente o tempo foi ajustado, pois as fibras da madeira têm um escoamento menor do que o polímero virgem. Nos processos de fabricação do compósito, tanto na extrusora como na injetora, foi observado um odor específico da madeira.

\subsection{Microscopia eletrônica de varredura}

A microscopia eletrônica de varredura (MEV) foi realizada no Laboratório de Anelasticidade e Biomateriais da Faculdade de Ciências da UNESP de Bauru. Para a caracterização morfológica dos compósitos através da microscopia eletrônica de varredura, foram analisadas as superfícies dos corpos de prova submetidos ao teste de tração, e as amostras analisadas foram cortadas no tamanho de $1 \mathrm{~cm}^{2}$.

Este procedimento visa avaliar os aspectos morfológicos do compósito, analisando a capacidade de aglutinação das fibras da madeira com o polímero e podem ser melhores visualizados da Figura 5(a) até Figura 5(e). Os aspectos de adesão do compósito $90 \% \mathrm{PP}+10 \% \mathrm{FM}$ podem ser visualizados na Figura 5(a), com a estrutura polimérica caracterizada por pontos circulares na micrografia. Observa-se ainda, na MEV, em ampliação de 7.250x visto na Figura 5(b), o detalhe da aglutinação das partes do compósito. 

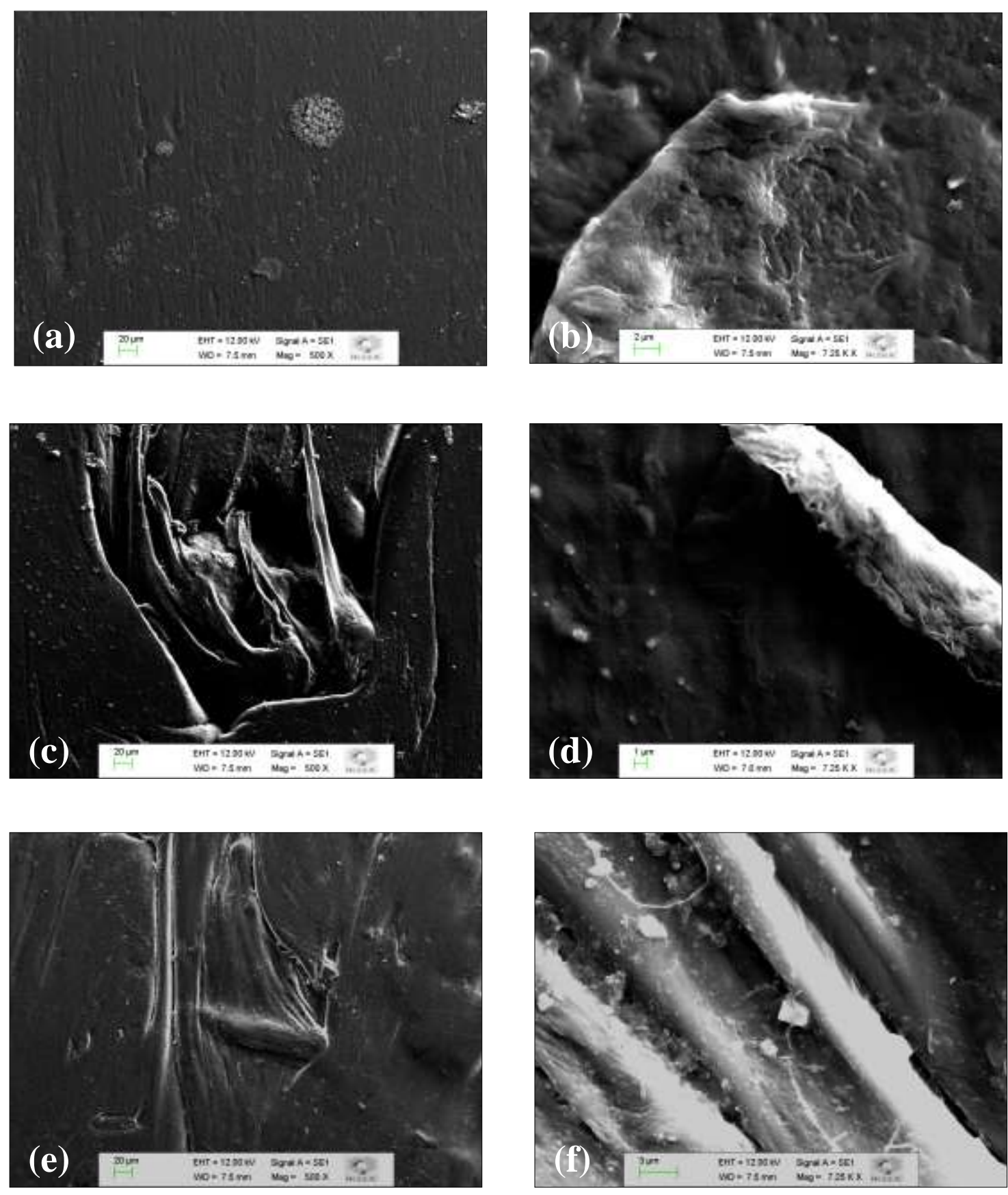

Figura 5: MEV dos compósitos de: 10\%FM em ampliação de (a) 500x e (b) 7.250x; 20\%FM em ampliação de (c) 500x e (d) 7.250x e 30\% FM em ampliação de (e) 500x e (f) 7.250x.

Na MEV do compósito de 20\%FM (Figura 5(c)), em ampliação de 500x, é possível visualizar a aderência das fibras de madeira com o polipropileno. Observa-se na Figura 5(f) MEV de ampliação em 7.250x, que o compósito com 30\%FM apresentou agregado de fibras. As imagens mostram claramente que os compósitos apresentaram adesão entre as fibras e o polímero, independente do percentual de material utilizado.

\section{CONCLUSÕES}

Um dos maiores desafios que podem ser encontrados na produção de compósitos plástico-madeira e que apresentam resultados satisfatórios nos ensaios físicos e mecânicos, segundo as normas relacionadas, é a adesão das fibras com a matriz polimérica, tendo em vista que muitos estudiosos fazem uso de agentes compatibilizantes na composição, a fim de proporcionar tal adesão. Entretanto, os resultados dos ensaios realizados 
nesta pesquisa mostraram que é possível a extrusão e a injeção do compósito com até $30 \% \mathrm{FM}$, sem nenhum aglutinante ou compatibilizante nas formulações, visto o uso do polímero reciclado que pode conter, em seu processamento primário, a adição de tais elementos compatibilizantes, tornando seu uso uma vantagem econômica, uma vez que pode ser desnecessário incorpora-los novamente na fabricação do compósito para a aglutinação das fibras com a matriz.

Nos testes de absorção de água e inchamento em espessura, pode-se verificar que, quanto maior a quantidade de partículas de madeira, maiores são os valores encontrados, porém de uma forma significativamente baixa, pois o polímero não absorve àgua (menor umidade).

Este trabalho demonstrou a viabilidade da produção de compósitos de plástico-madeira sem adição de compatibilizantes e outros aditivos. Utilizando-se de materiais reciclados, como o polímero e resíduos de madeira que seriam descartados pela maioria das indústrias madeireiras, por ser um resíduo de granulometria muito fina. Tais resíduos madeireiros poderiam estar sendo lançados na natureza ou queimados a céu aberto, contribuindo para o aumento da poluição.

Segundo MONDARDO [21], diversos estudos demonstraram que as fibras curtas melhoram a rigidez do produto final, porém prejudicam os resultados de resistência ao impacto. Fibras mais longas melhoram a resistência ao impacto, contudo, apresentam dificuldades de dispersão do polímero. Tal observação também foi verificada neste estudo, confirmando que os compósitos produzidos com fibras curtas, ou farinha de madeira, melhoram a rigidez, porém, diminuem a resistência ao impacto.

Em comparação com outras pesquisas, verifica-se que o uso de aditivos pode melhorar algumas propriedades, mas é preciso um estudo para verificar a viabilidade econômica do uso deste material já que a intenção é o aproveitamento de materiais reciclados. Visto que, nesta pesquisa, o PP usado foi proveniente de processo de reciclagem e não foram adicionados agentes compatibilizantes e aditivos, apresentando melhores resultados que o PP virgem usado em [19], para estudos futuros sugere-se a análise química do PP utilizado neste trabalho para averiguar tal fenômeno, visto que não foi um resultado esperado e os objetivos do trabalho não visavam uma análise química deste polímero reciclado.

\section{REFERÊNCIAS BIBLIOGRÁFICAS}

[1] MENEGHETTI, L. C. et al., "Verificação da possibilidade de variação das fibras formadoras do compósito nos sistemas PRF aplicados no reforço à flexão de fibras de concreto armado". Revista Ciência \& Engenharia, v.16, n. 1/2, 2007. Disponível em: http://www.seer.ufu.br/index.php /cieng/article/view/660/598. Acesso em novembro de 2012.

[2] NEWELL, J., Essentials of Modern materials Science and Engineering, Hoboken: John Wiley and Sons Ltd, 2009. Disponível em: http://www.lume.ufrgs.br/bitstream/handle/10183/27186/000763680.pdf? sequence $=1$ >. Acesso em fevereiro de 2012.

[3] CLEMONS, C.M., "Wood-Plastic Composites in the United States: The Interfacing of Two Industries", Forest Products Journal, pp. 10-18, June. 2002. Disponível em: http://www.fpl.fs.fed.us/documnts /pdf2002/clemo02b.pdf . Acesso em setembro 2010.

[4] RODOLFO JUNIOR, A., JOHN, V. M., "Desenvolvimento de PVC reforçado com resíduos de Pinus para substituir madeira convencional em diversas aplicações", Polímeros: Ciência e Tecnologia, v. 16, n. 1, PP. 1-11, 2006. Disponivel em:< http://www.scielo.br/scielo.php?script=sci_arttext\&pid=S010414282006000100005>. Acesso em setembro de 2010.

[5] MANRICH, S., Processamento de termoplásticos - rosca única, extrusão e matrizes, injeção e moldes, São Paulo, Artliber, 2005.

[6] NAJAFI, S. K., "Use of recycled plastics in wood plastic composites - a review", Waste Management,n.33, pp. 1898-1905, june 2013. Disponivel em:< http://www.medsci.cn/sci/show_paper.asp?id= 85fd5594600>. Acesso em junho de 2014.

[7] ECKERT, C., "Opportunities for natural fibers in plastic composites", 2000, apud JEONG, G. Y., Fracture behavior of wood plastic composite (WPC), Tese, Graduate School of the Louisiana State University and Agricultural and Mechanical College, Chonnam National University, 2004.

[8]STARK, N. Influence of moisture absorption on mechanical properties of wood flour-polypropylene composites. Journal of Thermoplastic Composite Materials, v.14, n.5, p.421-432, 2001. Disponível em :< http://www.scielo.br/scielo.php?script=sci_nlinks\&ref=000223\&pid=S1678-8621201300030001700024\&lng $=\mathrm{PT}>$. Acesso em junho de 2014 . 
[9] JOSEPH, P.V. et al. Environmental effects on the degradation behaviour of sisal fibre reinforced polypropylene composites. Composites Science and Technology, v.62, p.1357-72, 2002. Disponível em:< http://www.researchgate.net/publication/223430330_Environmental_effects_on_the_degradation_behaviour_ of_sisal_fibre_reinforced_polypropylene_composit es>. Acesso em junho de 2014 .

[10] PENDLETON, D.E et al. Durability of an extruded HDPE/wood composite. Forest Products Journal, v.52, n.6, p.21-27, 2002. Disponível em:< http://128.104.77.228/documnts/pdf2002/pendl02a.pdf >. Acesso em junho de 2014.

[11] STARK, N.M et al. Heat release rate of wood-plastic composites. Sampe Journal, v.33, n.5, p.26-31, 1997. Disponível em:< http://www.fpl.fs.fed.us/documnts/pdf1997/stark97a .pdf>. Acesso em junho de 2012.

[12] EMBRAPA. Sistemas de Produção, ISSN 1678-8281, versão eletrônica Nov./2005. Disponível em: http://sistemasdeproducao.cnptia.embrapa.br/Fontes/Pinus/Cultivodo Pinus/03_1_pinus_elliotti.htm. Acesso em outubro de 2013.

[13] American Society for Testing and Materials. ASTM D638-10: Standard Test Method for Tensile Properties of Plastics. 2010.

[14] American Society for Testing and Materials. ASTM D570-98: Standard Test Method for Water Absorption of Plastics, 1998.

[15] American Society for Testing and Materials. ASTM D-1238-10: Standard Test Method for Melt Flow Rates of Thermoplastics by Extrusion Plastometer, 2010.

[16] American Society for Testing and Materials. ASTM D256-00: Standard Test Methods for Determining the Izod Pendulum Impact Resistance of Plastics, 2000.

[17] YAMAJI, F. M., "Produção de compósito plástico-madeira a partir de resíduos da indústria madeireira”, Tese, UFPR, Curitiba, PR, Brasil, 2004. Disponível em:< http://www.floresta.ufpr.br/posgraduacao/defesas/pdf_dr/2004/t174_0203-D.pdf.> Acesso em fevereiro 2010.

[18] HILLIG, E. et. al., "Caracterização de compósitos produzidos com polietileno de alta densidade e serragem da indústria moveleira", Revista Árvore, v.32, n.2, pp.299-310, 2008. Disponível em: http://www.scielo.br/pdf/rarv/v32n2/ a13v32n2.pdf. Acesso em maio 2012.

[19] CORREA, C. A. et al., "Compósitos termoplásticos com madeira”, Polímeros - Ciência e Tecnologia, v.13, n.3, pp.154-165, 2003.

[20] CARASCHI, J. C., LEÃO, A. L., “Avaliação das propriedades mecânicas dos plásticos reciclados provenientes de resíduos sólidos urbanos”. Acta Scientiarum Maringá, v. 24, n. 6, pp. 1599-1602, 2002. Disponível em: http://eduemojs.uem.br/ojs/ index.php/ActaSciTechnol/article/view/2462/1553. Acesso em: novembro de 2012.

[21] MONDARDO, F. H. Compósitos de polipropileno e farinha de madeira. Dissertação - Instituto de Química, Universidade Federal do Rio Grande do Sul, Porto Alegre, 2006. Disponível em:< http://www.lume.ufrgs.br/handle/10183/27186>. Acesso em abril de 2012. 